\title{
INVERSE LIMITS ON GRAPHS AND MONTOTONE MAPPINGS
}

\author{
BY
}

\author{
J. W. ROGERS, JR.
}

\begin{abstract}
In 1935, Knaster gave an example of an irreducible continuum (i.e. compact connected metric space) $K$ which can be mapped onto an arc so that each point-preimage is an arc. The continuum $K$ is chainable (or arc-like). In this paper it is shown that every one-dimensional continuum $M$ is a continuous image, with arcs as point-preimages, of some onedimensional continuum $M^{\prime}$. Moreover, if $M$ is $G$-like, for some collection $G$ of graphs, then $M^{\prime}$ can be chosen to be $G$-like. A corollary is that every chainable continuum is a continuous image, with arcs as point-inverses, of a chainable (and hence, by a theorem of Bing, planar) continuum. These investigations give rise to the study of certain special types of inverse limit sequences on graphs.
\end{abstract}

1. Introduction. If $P$ is a proper subset of a continuum $M$, let $\mathscr{E}(M-P)$ denote the collection to which $e$ belongs if and only if $e$ is the closure of a component of $M-P$. Then $G$ is a graph if and only if $G$ is a continuum (i.e. a compact connected metric space) and there exists a finite proper subset $P$ of $G$ (called a partition of $G$ ) such that $\mathcal{E}(G-P)$ is a finite collection, each element of which is an arc with both endpoints in $P$, and no two elements of which have two points in common. (Hence every graph is homeomorphic to the space of a one-dimensional simplicial complex.) The elements of $\tilde{E}(G-P)$ are called edges of the partition $P$ of $G$. Clearly, each edge of $P$ has only its endpoints in $P$, and if two edges intersect, their intersection consists of a single common endpoint of both. A finite subset of $G$ that contains a partition $P$ of $G$ is called a refinement of $P$, and is itself a partition of $G$.

If $P_{1}$ and $P_{2}$ are partitions of graphs $G_{1}$ and $G_{2}$ respectively, a map $f$ : $G_{1} \rightarrow G_{2}$ is simplicial relative to $\left(P_{1}, P_{2}\right)$ if and only if $f\left(P_{1}\right) \subset P_{2}$, and the restriction of $f$ to any edge of $P_{1}$ is either constant or a homeomorphism onto an edge of $P_{2}$. The map $f: G_{1} \rightarrow G_{2}$ is simplicial if and only if it is simplicial relative to some partitions of $G_{1}$ and $G_{2}$.

A map is light if and only if each point-preimage is totally disconnected. It is easy to see that $f: G_{1} \rightarrow G_{2}$ is light and simplicial relative to the partitions $P_{1}$ and $P_{2}$ of $G_{1}$ and $G_{2}$, respectively, if and only if the restriction of $f$ to each

Presented to the Society, November 20, 1971; received by the editors January 10, 1972. AMS (MOS) subject classifications (1970). Primary 54B25, 54C10; Secondary 54F 20. Key words and phrases. Monotone map, decomposition into arcs, simplicial inverse limit, $G$-like continuum.

Copyright $\odot 1973$, American Mathematical Society 
edge of $P_{1}$ is a homeomorphism onto an edge of $P_{2}$; moreover, if $f$ is light and simplicial, then each point-preimage is in fact finite.

Denote by $d(x, y)$ the distance from the point $x$ to the point $y$; by $D(H)$ the diameter of the point set $H$; by $\bar{H}$ its closure. If $G$ is a collection of point sets, $G^{*}$ denotes their union; if $s$ is an arc with endpoints $x$ and $y$, then Seg $(s)=$ $s-\{x, y\}$ is called the segment of $s$.

For an inverse limit sequence $\left\{X_{i}, f_{i}\right\}$, we require that $f_{i}$ map $X_{i+1}$ onto $X_{i}$, and denote by $\pi_{i}$ the projection map from the limit onto $X_{i}$. As a matter of convention, we take metrics for the coordinate spaces so that $D\left(X_{i}\right) \leq 1$ for each $i$. The metric for the limit is defined by

$$
d(x, y)=\sum_{i=1}^{\infty} d\left(\pi_{i}(x), \pi_{i}(y)\right) \cdot 2^{-i} .
$$

If $G$ is a collection of graphs, then a continuum $M$ is said to be $G$-like if and only if, for each $\epsilon>0$, there is an $\epsilon$-map (i.e. point-preimages having diameter $<\epsilon$ ) from $M$ onto an element of $G$. From [5], the $G$-like continua are precisely the continua homeomorphic to the limit of an inverse sequence with coordinate spaces in $G$.

The inverse limit sequence $\left\{G_{i}, f_{i}\right.$ on graphs (i.e. $G_{i}$ is a graph for each $i$ ) is said to be light (or simplicial) if and only if $f_{i}$ is light (or simplicial) for each i. $\left\{G_{i}, P_{i}, f_{i}\right\}$ is called a uniformly simplicial inverse limit sequence on graphs if and only if, for each $i, G_{i}$ is a graph, $P_{i}$ is a partition of $G_{i}$, and $f_{i}$ is a map from $G_{i+1}$ onto $G_{i}$ which is simplicial relative to $\left(P_{i+1}, P_{i}\right)$.

In $\$ 2$, we show that every one-dimensional continuum is homeomorphic to the limit of a light simplicial inverse limit sequence on graphs. This sequence need not be uniformly simplicial, however. In $\$ 3$, we study uniformly simplicial inverse limits on graphs, characterizing their limits. Not every one-dimensional continuum is such a limit, since each such limit is a union of arcs, which disqualifies, for example, the pseudo-arc. On the other hand, several well-known continua are shown to be such limits, including the dyadic solenoid.

In $\$ 4$, we apply these ideas to the problem concerning monotone mappings discussed in the abstract.

\section{Light simplicial inverse limits.}

Lemma 1. Suppose $x$ and $y$ are points of the graph $G$, and $a$ and $b$ are endpoints of the arc $s$. Then there is a light simplicial map $g$ from $s$ onto $G$ such that $g(a)=x$ and $g(b)=y$.

Proof. Let $P$ denote a partition of $G$. There exists a finite sequence $s(1)$, $s(2), \ldots, s(n)$ of arcs in $G$ with $x$ as one endpoint, each of which is a union of 
edges of $P$, such that the other endpoint of $s(n)$ is $y$, and $\bigcup_{i=1}^{n} s(i)=G$. Let $w_{0}=a, w_{1}, w_{2}, \ldots, w_{2 n-1}=b$ denote a sequence of points in the indicated order along $s$. For each odd \{even\} positive integer $i<2 n$, let $b_{i}$ denote a homeomorphism from the arc $\left[w_{i-1}, w_{i}\right]$ onto $s((i+1) / 2)\{s(i / 2)\}$ such that $b_{i}\left(w_{i-1}\right)=x$ $\left\{b_{i}\left(w_{i}\right)=x\right\}$. Define $g$ such that if $c \in\left[w_{i=1}, w_{i}\right]$, then $g(c)=b_{i}(c)$. Clearly, $g$ is both light and simplicial, since each $b_{i}$ is, and $g(s) \supset \bigcup_{i=1}^{n} s(i)=G$.

Theorem 1. Suppose $\epsilon>0$, and $f$ is a map from the graph $G$ onto the graph $G^{\prime}$. Then there is a light simplicial map $g$ from $G$ onto $G^{\prime}$ such that if $x \in G$, then $d(f(x), g(x))<\epsilon$.

Proof. Let $P^{\prime}$ denote a partition of $G^{\prime}$ such that each edge of $P^{\prime}$ has diameter $<\epsilon / 4$. There exists a partition $P$ of $G$ and a map $g^{\prime}$ from $G$ into $G^{\prime}$ such that (1) $g^{\prime}$ is simplicial relative to $\left(P, P^{\prime}\right)$, (2) if $x \in G$ and $f(x) \in P^{\prime}$, then $g^{\prime}(x)=f(x)$, and (3) if $x \in G$ and $f(x)$ lies in the interior of an edge $s$ of $P^{\prime}$, then $g^{\prime}(x) \in s$ [3, Theorem 7.3, p. 64]. From condition (2), $g^{\prime}(P)=P^{\prime}$, and from (3), if $x \in G, d\left(f(x), g^{\prime}(x)\right)<\epsilon / 4$. Still, $g^{\prime}(G)$ need not be $G^{\prime}$, and $g^{\prime}$ need not be light.

Suppose $s$ is an edge of $G$ with endpoints $a$ and $b$. Then either $g^{\prime}(a)=$ $g^{\prime}(b)$, or $g^{\prime}(a)$ and $g^{\prime}(b)$ are the endpoints of the edge $g^{\prime}(s)$ of $G^{\prime}$. In any case, let $U$ denote the union of all the edges of $G^{\prime}$ with either $g^{\prime}(a)$ or $g^{\prime}(b)$ as an endpoint. Since $U$ is a graph, there is a light simplicial map $g_{s}$ from $s$ onto $U$ such that $g_{s}(a)=g^{\prime}(a)$ and $g_{s}(b)=g^{\prime}(b)$, by Lemma 1. Clearly, $D(U)<3 \cdot \epsilon / 4$, so that if $x \in s$,

$$
d\left[f(x), g_{s}(x)\right] \leq d\left[f(x), g^{\prime}(x)\right]+d\left[g^{\prime}(x), g_{s}(x)\right]<\epsilon / 4+3 \cdot \epsilon / 4=\epsilon .
$$

Hence, if we define $g(x)$, for each $x \in G$ to be $g_{s}(x)$, for any edge $s$ of $G$ that contains $x$, then $d(f(x), g(x))<\epsilon$, and $g$ is clearly both light and simplicial. Moreover, since $g^{\prime}(P)=P^{\prime}$, and for each edge $s$ of $G, g(s)$ contains every edge of $G^{\prime}$ that intersects $g^{\prime}(s), g$ throws $G$ onto $G^{\prime}$.

Theorem 2. Every one-dimensional continuum, $M$, is bomeomorpbic to the limit of a light simplicial inverse limit sequence $\left\{G_{i}, f_{i}\right\}$ on graphs. Moreover, if $M$ is $G$-like for some collection $G$ of graphs, then $G_{i}$ may be chosen from $G$ for each $i$.

Proof. It is well known that every one-dimensional continuum is homeomorphic to the limit of an inverse sequence on graphs [5]. Theorem 2 follows from this fact, Theorem 1, and Brown's approximation theorem for inverse limits [2, Theorem 3, p. 481].

3. Light uniformly simplicial inverse limits. While Theorem 2 shows that 
every one-dimensional continuum can be obtained from a light simplicial inverse limit sequence on graphs, the limits of light uniformly simplicial inverse sequences are far more restricted. It is the purpose of this section to bring out some of their properties in order to characterize them.

Theorem 3. Suppose (1) $\left\{G_{i}, P_{i}, g_{i}\right\}$ is a light uniformly simplicial inverse limit sequence, (2) $B$ is the collection of all sequences $\left\{s_{i}\right\}$ where, for each $i$, $s_{i}$ is an edge of $P_{i}$ and $s_{i}=g_{i}\left(s_{i+1}\right)$, (3) for each $\left\{s_{i}\right\} \in B, \lambda\left\{s_{i}\right\}=$ $\bigcap_{i=1}^{\infty} \pi_{i}^{-1}\left(s_{i}\right)$, and (4)A is the collection of sets $\lambda\left\{s_{i}\right\}$ for all sequences $\left\{s_{i}\right\}$ in $B$. Then (a) each element of $A$ is an arc, (b) for each $i$, the restriction of $\pi_{i}$ to any element of $A$ is a bomeomorphism onto an edge of $G_{i}$, and (c) $M=A^{*}$.

Proof. Suppose $a=\lambda\left\{s_{i}\right\} \in A$. Then $\alpha$ is the limit of the inverse sequence $\left\{s_{i}, g_{i} \mid s_{i}\right\}$. Since $f_{i}$ is light and simplicial for each $i$, and $s_{i}$ is an edge of $G_{i}$, $g_{i} \mid s_{i}$ is a homeomorphism. But if each of the bonding maps is a homeomorphism, then so are the projection maps, $\pi_{i} \mid \alpha$, which proves (b). But then $\alpha$ is homeomorphic to $\pi_{1}(\alpha)=s_{1}$, an arc, which proves (a).

For (c), suppose $p\left(p_{1}, p_{2}, \ldots\right)$ is a point of $M_{\text {. If }} p_{1}$ is not in $P_{1}$, then by induction $p_{i}$ is not in $P_{i}$ for any $i$, and there is only one edge, $s_{i}$ of $G_{i}$ that contains $p_{i}$. For each $i, g_{i}\left(s_{i+1}\right)$ is some edge of $G_{i}$ that contains $g_{i}\left(p_{i+1}\right)=$ $p_{i}$, but $s_{i}$ is the only such edge, so $s_{i}=g_{i}\left(s_{i+1}\right)$. Hence $\left\{s_{i}\right\} \in B$, and $p \in$ $\lambda\left\{s_{i}\right\} \in A$.

If, on the other hand, $p_{1} \in P_{1}$, then, again by induction, $p_{i} \in P_{i}$ for each $i$. Now, for each $i$, let $H_{i}$ denote the collection of all edges of $G_{i}$ with $p_{i}$ as an endpoint. Clearly if $s \in H_{j}$ and $i<j$, then $g_{i j}(s) \in H_{i}$. Hence some element $s_{1}$ of the finite set $H_{1}$ is the image under $g_{1 j}$ of an element of $H_{j}$ for infinitely many (and hence clearly for all) positive integers $j>1$. For each $j>1$, let $H_{j}^{1}$ denote the set to which $s$ belongs if and only if $s \in H_{j}$ and $g_{1 j}(s)=s_{1}$. Continuing by induction, construct sequences $\left\{s_{i}\right\}$ and $\left\{H_{j}^{i}\right\}(i<j)$ such that $s_{i} \in H_{i}^{i-1}$ and, for each $j>i, s \in H_{j}^{i}$ if and only if $s \in H_{j}^{i-1}$ and $g_{i j}(s)=s_{i}$ (the finiteness of $H_{i}^{i-1}$ assures the existence of an edge $s_{i}$ which is the image under $g_{i j}$ of some element of $H_{j}^{i-1}$, for each $\left.j>i\right)$. Then $\left\{s_{i}\right\} \in B$, and $p \in \lambda\left\{s_{i}\right\} \in A$.

Theorem 4 is useful for generating examples of limits of light uniformly simplicial inverse systems. We precede it with a lemma.

Lemma 2. Suppose $P$ and $P_{0}$ are partitions of the graphs $G$ and $G_{0}$, respectively, $f$ is a light simplicial map from $G$ onto $G_{0}$ relative to $\left(P, P_{0}\right), Q_{0}$ is a refinement of $P_{0}$, and $Q=f^{-1}\left(Q_{0}\right)$. Then $Q$ is a refinement of $P$, and $f$ is simplicial relative to $\left(Q, Q_{0}\right)$.

Proof. Since $f$ throws $G$ onto $G_{0}, f^{-1}\left(P_{0}\right)=P$, and since $f$ is light and simplicial, $f^{-1}\left(Q_{0}\right)$ is finite. Hence $Q=f^{-1}\left(Q_{0}\right) \supset f^{-1}\left(P_{0}\right)=P$, and $Q$ is a 
refinement of $P$. Moreover, each edge $s$ of $Q$ lies in an edge of $P$ on which $f$ is a homeomorphism. Hence $f(s)$ is an arc in $G_{0}$ with only its endpoints in $Q_{0}$, i.e. $f(s)$ is an edge of $Q_{0}$. So the restriction of $f$ to any edge of $Q$ is a homeomorphism onto an edge of $Q_{0}$ and $f$ is simplicial relative to $\left(Q, Q_{0}\right)$.

Theorem 4. Suppose $f$ is a map from the graph $G$ onto itself, and $P_{1}$ is a partition of $G$ such that $P_{2}=f^{-1}\left(P_{1}\right)$ is a refinement of $P_{1}$, and $f$ is light and simplicial relative to $\left(P_{2}, P_{1}\right)$. Then $\left\{G_{i}, P_{i}, f_{i}\right\}$ is a light uniformly simplicial inverse sequence, where for each $i, G_{i}=G, f_{i}=f$, and $P_{i+1}=f^{-1}\left(P_{i}\right)$.

Proof. By hypothesis, $f_{1}$ is simplicial relative to $\left(P_{2}, P_{1}\right)$. Since $P_{2}$ is a refinement of $P_{1}, P_{3}=f^{-1}\left(P_{2}\right)$ is a refinement of $P_{2}$ by Lemma 2 , and $f_{2}=f$ is simplicial relative to $\left(P_{3}, P_{2}\right)$. The proof continues by induction.

Several well-known continua are limits of inverse sequences with a single bonding map that satisfies the hypothesis of Theorem 4. For example, if $I$ denotes the unit interval, and $f: I \rightarrow I$ is defined by $f(x)=2 x$ if $0 \leq x \leq 1 / 2$ and $f(x)=$ $(3 / 2)-x$ if $1 / 2 \leq x \leq 1$, then the limit of the inverse sequence $\{I, f\}$ (i.e. each coordinate space is $l$, and each bonding map is $f)$ is the $\sin (1 / x)$-continuum, and $P_{1}=\{0,1 / 2,1\}$ satisfies the hypothesis of Theorem 4. If $g(x)=2 x(0 \leq x \leq$ $1 / 2)$ and $g(x)=2-2 x(1 / 2 \leq x \leq 1)$, then the limit of the inverse sequence $\{I, g\}$ is a well-known planar indecomposable continuum, and again $P_{1}=\{0,1 / 2,1\}$ satisfies the theorem. Finally, if $C$ denotes the unit circle in the complex plane, and $s(z)=z^{2}$ for each complex number $z$ in $C$, then $\lim \{C, s\}$ is the dyadic solenoid, and $P_{1}=\{1,-1\}$ satisfies the theorem. Hence each of these continua is a limit of a light uniformly simplicial inverse sequence. We are now ready to characterize all the limits of such sequences.

Theorem 5. In order that the continum $M$ be bomeomorpbic to the limit of a light uniformly simplicial inverse sequence on graphs, it is both necessary and sufficient that there exist a map $\pi$ from $M$ onto $[0,1]$ such that (1) $V=$ $\pi^{-1}(\{0,1\})$ is totally disconnected, and (2) if a $\in \mathcal{E}(M-V)$, then $\pi \mid \alpha$ is a bomeomorphism onto $[0,1]$.

Proof of necessity. We assume that $M$ is homeomorphic to such a sequence $\left\{G_{i}^{\prime}, P_{i}^{\prime}, f_{i}\right\}$. Our first object is to modify the inverse sequence so that $[0,1]$ is the first coordinate space. Then the first projection mapping will satisfy the requirements for $\pi$.

For each edge $e$ of $P_{2}^{\prime}$, let $x_{e}$ denote a particular nonendpoint of $e$, and let $X$ denote the union of the points $x_{e}$ for all edges $e$ of $P_{2^{\prime}}^{\prime}$ and $P_{2}=X \cup P_{2}^{\prime}$. Then $P_{2}$ is a refinement of $P_{2}^{\prime}$. Let $G_{1}=[0,1], G_{2}=G_{2}^{\prime}$, and $P_{1}=\{0,1\}$. Define $\phi: P_{2} \rightarrow P_{1}$ such that $\phi\left(P_{2}^{\prime}\right)=0$ and $\phi(X)=1$. Then $\phi$ is easily extended to a light simplicial map $g_{1}$ from $G_{2}$ onto $G_{1}$ relative to $\left(P_{2}, P_{1}\right)$. 
For each $n>1$, let $G_{n+1}=G_{n+1}^{\prime}$, and $P_{n+1}=f_{n}^{-1}\left(P_{n}\right)$. Then by Lemma 2, $P_{n+1}$ is a refinement of $P_{n+1}^{\prime}$, and $f_{n}$ is simplicial relative to $\left(P_{n+1}, P_{n}\right)$. So if $f_{n}=g_{n}$ for each $n>1$, then $\left\{G_{i}, P_{i}, g_{i}\right\}$ is a light uniformly simplicial inverse system with limit $M^{\prime}$ homeomorphic to $M$, since only the first coordinate space and bonding map have been altered.

We now show that the first projection map $\pi_{1}: M^{\prime} \rightarrow G_{1}=[0,1]$ has the required properties relative to $M^{\prime}$. First, $g_{i}$ is light for each $i$, so $\pi_{1}$ is light, and $\pi_{1}^{-1}(0)$ and $\pi_{1}^{-1}(1)$ are totally disconnected. For condition (2), we begin by letting $A$ denote the collection as defined in Theorem 3 relative to $\left\{G_{i}, P_{i}, g_{i}\right\}$, and $\alpha=\lambda\left\{s_{i}\right\}$ and $\alpha^{\prime}=\lambda\left\{s_{i}^{\prime}\right\}$ denote two elements of $A$. Hence, letting $V=$ $\pi_{1}^{-1}(\{0,1\})$, both Seg $(\alpha)$ and Seg $\left(\alpha^{\prime}\right)$ lie in $M^{\prime}-V$. Let $n$ denote the first positive integer $i$ such that $s_{i} \neq s_{i}^{\prime}$. Then $\operatorname{Seg}\left(s_{i}\right)$ and $G_{i}-s_{i}$ are mutually exclusive open subsets of $G_{i}$, and $\operatorname{Seg}\left(s_{i}^{\prime}\right) \subset G_{i}-s_{i}$. Hence $\pi_{i}^{-1}\left(\operatorname{Seg}\left(s_{i}\right)\right)$ and $\pi_{i}^{-1}\left(G_{i}-s_{i}\right)$ are mutually exclusive open subsets of $M^{\prime}-V$, containing $\operatorname{Seg}(a)$ and $\operatorname{Seg}\left(\alpha^{\prime}\right)$ respectively. We conclude that if $\alpha$ and $\alpha^{\prime}$ are elements of $A$, then $\operatorname{Seg}(\alpha)$ and $\operatorname{Seg}\left(\alpha^{\prime}\right)$ lie in different components of $M^{\prime}-V$. Hence Seg $(\alpha)$ is itself a component of $M^{\prime}-V$, for every point of $\left(M^{\prime}-V\right)-\operatorname{Seg}(\alpha)$ lies in the segment of some other arc of $A$. Hence the components of $M^{\prime}-V$ are precisely the segments of arcs of $A$, and the elements of $\mathcal{E}\left(M^{\prime}-V\right)$ are these arcs. The restriction of $\pi_{1}$ to each of these arcs is now a homeomorphism onto $[0,1]$ by Theorem 3.

Proof of sufficiency. Suppose $\pi$ is a map from $M$ onto $[0,1]$ satisfying the conditions of the theorem. Let $A=\pi^{-1}(0)$ and $B=\pi^{-1}(1)$ and note that it follows from the conditions of the theorem that if $x$ is a point of $M-V$, then $\alpha_{x}$, the closure of the component of $M-V$ that contains $x$, is an arc with one endpoint in $A$ and the other in $B$. We first consider the case in which it is true that

(Assumption Z) If $x$ and $y$ lie in $M-V$, and both endpoints of $a_{x}$ are endpoints of $a_{y}$, then $a_{x}=a_{y}$.

Now, since $A \subset V, A$ is totally disconnected; so there is a sequence $\left\{A_{i}\right\}$ such that, for each $i$, (1) $A_{i}$ is a finite collection of mutually exclusive closed subsets of $A$, each of diameter $<2^{-i}$, (2) $A_{i}^{*}=A$, and (3) each element of $A_{i+1}$ lies in some element of $A_{i}$. There is a sequence $\left\{B_{i}\right\}$ for $B$ with similar properties.

Suppose $i$ is a positive integer. Let $\phi_{i}$ denote a one-to-one map from the collection $A_{i} \cup B_{i}$ onto a finite subset $V_{i}$ of $E^{3}$ of diameter 1 , such that no three points of $V_{i}$ are collinear, and no four are coplanar. Let $e_{i}: M \rightarrow E^{3}$ denote the map such that (1) if $a \in A_{i}, e_{i}(a)=\phi_{i}(a)$, (2) if $b \in B_{i}, e_{i}(b)=\phi_{i}(b)$, and (3) if $x \in \mathcal{E}(M-V)$ and $a_{x}$ has endpoints in $a \in A_{i}$ and $b \in B_{i}$, respectively, then $e_{i}(x)$ is the point between $\phi_{i}(a)$ and $\phi_{i}(b)$ given by $t \phi_{i}(b)+(1-t) \phi_{i}(a)$, where $t=\pi(x) \in[0,1]$. Let $G_{i}=e_{i}(M)$, a graph in $E^{3}$. 
Now, if $i$ is a positive integer and $a \in A_{i+1}$ [or $b \in B_{i+1}$ ], then there is only one element $v(a)$ [or $v(b)]$ of $A_{i}$ [or $B_{i}$, that contains $a$ [or $b$ ]. Hence there is a linear map $g_{i}$ from $G_{i+1}$ onto $G_{i}$ such that if $x \in M-V$, and $\alpha_{x}$ has endpoints in $a \in A_{i+1}$ and $b \in B_{i+1}$ respectively, and $y \in G_{i+1}$ is the point $e_{i+1}(x)=t \phi_{i+1}(b)+(1-t) \phi_{i+1}(a)$, where $t=\pi(x)$, then $g_{i}(y)$ is the point $e_{i}(x)=$ $t \phi_{i}[v(b)]+(1-t) \phi_{i}[v(a)]$ of $G_{i}$. So, $e_{i}=g_{i} e_{i+1}$, and it is not difficult to see that $\left\{G_{i}, V_{i}, g_{i}\right\}$ is a light uniformly simplicial inverse sequence on graphs.

We now show that $M$ and $M^{\prime}$, the limit of the inverse sequence $\left\{G_{i}, V_{i}, f_{i}\right\}$ are homeomorphic. For each $x \in M$, let $b(x)=\left(e_{1}(x), e_{2}(x), \ldots\right)$. Then for each $i, e_{i}(x) \in G_{i}$, and $e_{i}(x)=g_{i} e_{i+1}(x)$, so that $b(x) \in M^{\prime}$.

If $x \in M$, and $O$ is an open set in $M^{\prime}$ containing $b(x)$, then there is a positive integer $i$ and an open set $R$ in $G_{i}$ containing $\pi_{i} b(x)=e_{i}(x)$ such that $\pi_{i}^{-1}(R) \subset O$. Let $O^{\prime}=e_{i}^{-1}(R)$. Then $O^{\prime}$ is open in $M$ and contains $x$, and

$$
b\left(O^{\prime}\right) \subset \pi_{i}^{-1}\left[e_{i}\left(O^{\prime}\right)\right]=\pi_{i}^{-1}[R] \subset O .
$$

Hence $b$ is continuous.

That $h(M)=M^{\prime}$ follows from the fact that for each $i, \pi_{i}[b(M)]=e_{i}(M)=G_{i}$, and no closed proper subset of $M^{\prime}$ projects onto every coordinate space.

Finally, $b$ is reversible, for suppose $x$ and $y$ are distinct points of $M$. If $x \in A$, there is a positive integer $i$ such that $x \in a \in A_{i}$, but $y \notin a$. So $e_{i}(x)=$ $\phi_{i}(a) \neq e_{i}(y)$, and $b(x) \neq b(y)$. The argument is similar if either $x$ or $y$ lies in $A \cup B$. So suppose neither $x$ nor $y$ lies in $V$. If $\alpha_{x}=a_{y}$, then $e_{1}(x) \neq e_{1}(y)$ since $e_{1} \mid \alpha_{x}$ is a homeomorphism, and again, $b(x) \neq b(y)$. If $\alpha_{x} \neq \alpha_{y}$, let $c_{x}$ $\in A$ [or $\left.c_{y} \in B\right]$ and $d_{x} \in B\left[d_{y} \in B\right]$ denote the endpoints of $\alpha_{x}\left[\alpha_{y}\right]$. By Assumption $Z$, either $c_{x} \neq c_{y}$ or $d_{x} \neq d_{y}$; suppose $c_{x} \neq c_{y}$. Then for some $i$, $c_{x} \in a_{x} \in A_{i}$ and $c_{y} \in a_{y} \in A_{i}$, and $a_{x} \neq a_{y}$. Hence

$$
e_{i}\left(c_{x}\right)=\varnothing_{i}\left(a_{x}\right) \neq \varnothing_{i}\left(a_{y}\right)=e_{i}\left(c_{y}\right) \text {. }
$$

But $e_{i}(x)$ lies between $e_{i}\left(c_{x}\right)$ and $e_{i}\left(d_{x}\right)$ and $e_{i}(y)$ lies between $e_{i}\left(c_{y}\right)$ and $e_{i}\left(d_{y}\right)$. Since no three of the points of $e_{i}\left(\left\{c_{x}, c_{y}, d_{x}, d_{y}\right\}\right)$ are collinear, and no four coplanar, $e_{i}(x) \neq e_{i}(y)$, and so $b(x) \neq b(y)$.

This completes the argument if Assumption $\mathrm{Z}$ is satisfied. If it is not satisfied, define $g: I \rightarrow I$ such that $g(x)=2 x(0 \leq x \leq 1 / 2)$ and $g(x)=2-2 x$ $(1 / 2 \leq x \leq 1)$. Let $\pi^{\prime}=g \pi, V^{\prime}=\pi^{\prime-1}(\{0,1\})$ and note that $\pi^{\prime}$ and $V^{\prime}$ satisfy the conditions required of $\pi$ and $V$ in the statement of the theorem. For each $x \in M-V^{\prime}$, let $a_{x}^{\prime}$ denote the element of $\mathscr{E}\left(M-V^{\prime}\right)$ that contains $x_{\text {. }}$ It is easily verified that if $y \in M-V$, and $u=\pi^{-1}(3 / 4) \cap \alpha_{y}$, and $v=\pi^{-1}(1 / 4) \cap \alpha_{y}$, then $\alpha_{y}=\alpha_{u}^{\prime} \cup \alpha_{v}^{\prime}, \alpha_{u}^{\prime} \cap \alpha_{v}^{\prime}=\pi^{-1}(1 / 2) \cap \alpha_{y}$, and if $x$ and $y$ are points of $M-V$ and $a_{x} \neq a_{y}$, then $\pi^{-1}(1 / 2) \cap a_{x} \neq \pi^{-1}(1 / 2) \cap a_{y}$. It follows quickly then that if $x$ 
and $y$ lie in $M-V^{\prime}$, and both endpoints of $\alpha_{x}^{\prime}$ are endpoints of $\alpha_{y}^{\prime}$, then $\alpha_{x}^{\prime}=\alpha_{y}^{\prime}$. That is, Assumption $Z$ is satisfied for $\pi^{\prime}$ and $V^{\prime}$. The proof is now similar to the first case, using $\pi^{\prime}$ and $V^{\prime}$ in place of $\pi$ and $V$.

Note that it follows from this characterization that each arc-component of the limit of a light uniformly simplicial inverse sequence is nondegenerate. This fact can be used to generate a number of continua which are not such limits.

4. An application to the theory of monotone mappings. The object of this section is to use the ideas developed in the previous two sections to prove the following theorem.

Theorem 6. If $M$ is a one-dimensional continuum, then there exists a onedimensional continuum $M^{\prime}$ and a map $\theta$ from $M^{\prime}$ onto $M$ under which each pointpreimage is an arc. Moreover, if $M$ is $G$-like for some collection $G$ of graphs, then $M^{\prime}$ can be chosen to be G-like.

Proof. We take $M$ to be the limit of a light simplicial inverse sequence $\left\{G_{i}, f_{i}\right\}$ as in Theorem 2, picking $G_{i}$ from $G$, for each $i$, if $M$ is $G$-like. To avoid later confusion, we denote the projection map from $M$ onto $G_{i}$ by $\pi_{i}^{f}$. Using Lemma 2, we construct sequences $\left\{P_{i}\right\}$ and $\left\{P_{i}^{\prime}\right\}$ such that for each $i$,

(1) $P_{i}^{\prime}$ is a refinement of the partition $P_{i}$ of $G_{i}$,

(2) $f_{i}$ is simplicial relative to $\left(P_{i+1}, P_{i}^{\prime}\right)$,

(3) if $s$ is an edge of $P_{i}$, then

(a) $P_{i}^{\prime} \cap s$ consists of an even number $\geq 8$ of points,

(b) at most one endpoint of $s$ is of order $>2$ in $G_{i}$,

(c) no point of $P_{i}$ is adjacent to both endpoints of $s$, and

(d) if $j<i$, then $D\left[f_{j i}(s)\right]<2^{j-i}$.

For each $i>0$ and $n>0$, let $C_{i}(n)$ denote the collection of all subsets $L$ of $G_{i}$ such that if $x$ and $y$ are points of $L$, then there is a sequence $s_{1}, \ldots$, $s_{m}$, where $m \leq n$, of edges of $P_{i}$ such that $x \in s_{1}, y \in s_{m}$, and if $|j-k|=1$, then $s_{j}$ and $s_{k}$ are adjacent. Suppose $c \in C_{i}(n)$. Then if $j<i$, it follows from property (3d) above that $D\left[f_{j i}(c)\right] \leq n \cdot 2^{j-i}$. Hence, if $c^{\prime}=\left(\pi_{i}^{f}\right)^{-1}[c]$,

$$
\begin{aligned}
D\left[c^{\prime}\right] & \leq \sum_{j=1}^{\infty} D\left[\pi_{j}^{f}\left(c^{\prime}\right)\right] \cdot 2^{-j} \leq \sum_{j=1}^{j} D\left[f_{j i}(c)\right] \cdot 2^{-j}+\sum_{j=i+1}^{\infty}(1) \cdot 2^{-j} \\
& \leq \sum_{j=1}^{i}\left[\left(n \cdot 2^{j-i}\right) \cdot 2^{-j}\right]+2^{-i} \\
& =\sum_{j=1}^{i}\left(n \cdot 2^{-i}\right)+2^{-i}=i n 2^{-i}+2^{-i}=2^{-i}(i n+1) .
\end{aligned}
$$

We have shown that 
$\left(A^{*}\right)$ if $c \in C_{i}(n)$, then $D\left[\left(\pi_{i}^{f}\right)^{-1}[c]\right] \leq 2^{-i}($ in +1$)$.

For each $i$, if $p \in P_{i}$ and $p$ is of order $>2$ in $G_{i}$, let $z(p)$ denote a definite point of $P_{i}$ adjacent to $p$. We now define a map $\phi_{i}: P_{i}^{\prime} \rightarrow P_{i}$. Suppose $s$ is any edge of $P_{i}$, and let $p_{1}, \cdots, p_{n}$ denote the points of $P_{i}^{\prime} \cap s$, in order along $s$, with $p_{1}$ the point of order $>2$ in $G_{i}$, if there is one. Then

(1) if $1 \leq j<n$ and $j$ is odd, $\phi_{i}\left(p_{j}\right)=p_{1}$,

(2) if $2<j \leq n$ and $j$ is even, $\phi_{i}\left(p_{j}\right)=p_{n}$,

(3) $\phi_{i}\left(p_{2}\right)=z\left(p_{1}\right)$ if the order of $p_{1}$ in $G_{i}>2$, and

(4) $\phi_{i}\left(p_{2}\right)=p_{n}$ if the order of $p_{1} \leq 2$.

Roughly, the points $p_{1}, \cdots, p_{n}$ are thrown atlernately to $p_{1}$ and $p_{n}$, except that if the order of $p_{1}>2$, then $p_{2}$ is thrown to $z\left(p_{1}\right)$. Define $\phi_{i}$ in this way on all the edges of $p_{i}$, and extend $\phi_{i}$ in the obvious manner to a light simplicial map $e_{i}$ from $G_{i}$ onto $G_{i}$ relative to $\left(P_{i}^{\prime}, P_{i}\right)$.

If $s$ is an edge of $P_{i}$ [or $P_{i}^{\prime}$ ] let $S(s)\left[S^{\prime}(s)\right]$ denote the union of all the edges of $P_{i}\left[P_{i}^{\prime}\right]$ that either are $s$, or are adjacent to $s$, and let $T(s)\left[T^{\prime}(s)\right]$ denote the collection of all edges of $P_{i}\left[P_{i}^{\prime}\right]$ that are either in $S(s)\left[S^{\prime}(s)\right]$ or adjacent to an edge in $S(s)\left[S^{\prime}(s)\right]$. If $s^{\prime}$ is an edge of $P_{i}^{\prime}$, let $E\left(s^{\prime}\right)$ denote the edge of $P_{i}$ that contains it. Then it follows from the properties of $e_{i}$, by a straightforward consideration of a number of different cases, that, for each $i$,

$\left(\mathrm{B}^{*}\right)$ if $s^{\prime}$ is an edge of $P_{i}^{\prime}$, then $e_{i}\left[T^{\prime}\left(s^{\prime}\right)\right]$ lies in the union of $E\left(s^{\prime}\right)$ and one edge of $P_{i}$ adjacent to $E\left(s^{\prime}\right)$, and

$\left(C^{*}\right)$ if $s^{\prime}$ is an edge of $P_{i}^{\prime}$, then $S^{\prime}\left(s^{\prime}\right) \subset S\left[e_{i}\left(s^{\prime}\right)\right]$.

For each positive integer $i$, let $g_{i}=\epsilon_{i} f_{i}$. Note that $g_{i}$ is light and simplicial relative to $\left(P_{i+1}, P_{i}\right)$. Also, if $s$ is an edge of $P_{i+1}$, and $s^{\prime}=f_{i}(s)$ (so that $s^{\prime}$ is an edge of $\left.P_{i}^{\prime}\right)$, then $f_{i}(s(s)) \smile s^{\prime}\left(s^{\prime}\right)$ and $f_{i}(T(s)) \smile T^{\prime}\left(s^{\prime}\right)$, since $f_{i}$ is simplicial. Hence,

$$
g_{i}[T(s)]=e_{i} f_{i}[T(s)] e_{i}\left[T^{\prime}\left(s^{\prime}\right)\right] .
$$

It now follows from $\left(B^{*}\right)$ that

$\left(D^{*}\right)$ if $s$ is an edge of $P_{i+1}$, then $g_{i}[T(s)]$ lies in the union of two adjacent edges of $P_{i}$.

Also, from $\left(C^{*}\right)$,

$$
f_{i}(S(s)) \subset S^{\prime}\left(s^{\prime}\right) \subset S\left[e_{i}\left(s^{\prime}\right)\right]=S\left[e_{i}\left(f_{i}(s)\right)\right]=S\left[g_{i}(s)\right],
$$

so that

$\left(\mathrm{E}^{*}\right)$ if $s$ is an edge of $P_{i+1}$, then $f_{i}[S(s)] \subset s\left[g_{i}(s)\right]$.

We are now ready to define $M^{\prime}$ as the limit of the light uniformly simplicial inverse sequence $\left\{G_{i}, P_{i}, g_{i}\right\}$. For each $i$, let $\pi_{i}^{8}$ denote the projection from $M^{\prime}$ onto $G_{i}$, and let $A$ denote the collection of arcs in $M^{\prime}$ as in Theorem 3. Suppose 
$\alpha=\lambda\left\{s_{i}\right\} \in A$. For each $i$, let $M_{i}(\alpha)=\left(\pi_{i}^{f}\right)^{-1}\left[S\left(s_{i}\right)\right] \subset M$. Since $S\left(s_{i}\right) \in C_{i}(3)$, it follows from $\left(A^{*}\right)$ that $D\left[M_{i}(\alpha)\right] \leq 2^{-i}(3 i+1)$, so that $\lim _{i \rightarrow \infty} D\left[M_{i}(\alpha)\right]=0$. Also, from $\left(\mathrm{E}^{*}\right), f_{i}\left[S\left(s_{i+1}\right)\right] \subset S\left[g_{i}\left(s_{i+1}\right)\right]=S\left(s_{i}\right)$, so

$$
M_{i+1}(\alpha)=\left(\pi_{i+1}^{f}\right)^{-1}\left[S\left(s_{i+1}\right)\right] \subset\left(\pi_{i}^{f}\right)^{-1}\left[f_{i}\left[S\left(s_{i+1}\right)\right]\right] \subset\left(\pi_{i}^{f}\right)^{-1}\left[S\left(s_{i}\right)\right]=M_{i}(\alpha) .
$$

Since $M$ is compact, these two facts imply that $\bigcap_{i=1}^{\infty} M_{i}(\alpha)$ consists of a single point, which we denote by $\theta^{\prime}(a)$.

We now establish the first of two statements about $\theta^{\prime}$.

$\left(\mathrm{F}^{*}\right)$ If $\alpha=\lambda\left\{s_{i}\right\} \in A$ and $\alpha^{\prime}=\lambda\left\{s_{i}^{\prime}\right\} \in A$, then $\theta^{\prime}(\alpha)=\theta^{\prime}\left(\alpha^{\prime}\right)$ if and only if, for each $i$, either $s_{i}=s_{i}^{\prime}$ or $s_{i}$ and $s_{i}^{\prime}$ are adjacent edges of $P_{i}$.

Suppose the latter is true. Then for each $i, S\left(s_{i}\right)$ intersects $S\left(s_{i}^{\prime}\right)$ and so $M_{i}(\alpha)$ intersects $M_{i}\left(\alpha^{\prime}\right)$, and $\lim _{i \rightarrow \infty} D\left[M_{i}(\alpha) \cup M_{i}\left(\alpha^{\prime}\right)\right]=0$. But

$$
\theta^{\prime}(\alpha) \cup \theta^{\prime}\left(\alpha^{\prime}\right) \subset \bigcap_{i=1}^{\infty}\left[M_{i}(\alpha) \cup M_{i}\left(\alpha^{\prime}\right)\right],
$$

which has diameter 0 , so $\theta^{\prime}(\alpha)=\theta^{\prime}\left(\alpha^{\prime}\right)$. On the other hand, if $\theta^{\prime}(\alpha)=\theta^{\prime}\left(\alpha^{\prime}\right)$, then $\pi_{i}^{f} \theta^{\prime}(a) \in S\left(s_{i}\right) \cap S\left(s_{i}^{\prime}\right)$ for each $i$. Suppose $i$ is any positive integer. Then, since $S\left(s_{i+1}\right)$ intersects $S\left(s_{i+1}^{\prime}\right)$, there is an edge $s$ of $P_{i+1}$ such that both $s_{i+1}$ and $s_{i+1}^{\prime}$ lie in $T(s)$. Hence $s_{i} \cup s_{i}^{\prime}=g_{i}\left(s_{i+1} \cup s_{i+1}^{\prime}\right) \subset g_{i}[T(s)]$ which, by $\left(D^{*}\right)$, lies in the union of two adjacent edges of $G_{i}$, and each of the edges $s_{i}$ and $s_{i}^{\prime}$ must be one of these edges, which completes the proof of $\left(\mathrm{F}^{*}\right)$.

$\left(G^{*}\right)$ If $p$ is a point of two elements, $\alpha$ and $\alpha^{\prime}$ of $A$, then $\theta^{\prime}(\alpha)=\theta^{\prime}\left(\alpha^{\prime}\right)$.

This is an immediate consequence of $\left(\mathrm{F}^{*}\right)$ since, for each $i$, the edge $s_{i}=$ $\pi_{i}^{g}(\alpha)$ and the edge $s_{i}^{\prime}=\pi_{i}^{g}\left(\alpha^{\prime}\right)$ have $\pi_{i}^{g}(p)$ in common.

We now define the function $\theta: M^{\prime} \rightarrow M$, making use of $\left(G^{*}\right)$. If $p \in M^{\prime}$, and $\alpha=\lambda\left\{s_{i}\right\}$ is any element of $A$ that contains $p$, then $\theta(p)=\theta^{\prime}(\alpha)=$ $\bigcap_{i=1}^{\infty}\left(\pi_{i}^{f}\right)^{-1}\left[S\left(s_{i}\right)\right]$. We conclude by showing that $\theta$ satisfies the requirements of the theorem.

First, $\theta$ is continuous, for suppose $\epsilon>0$, and $p\left(p_{1}, p_{2}, \ldots\right) \in M^{\prime}$. Then $p$ belongs to some element $\alpha=\lambda\left\{s_{i}\right\}$ of $A$. For each $i$, the interior, $S^{\alpha}\left(s_{i}\right)$, of $S\left(s_{i}\right)$ is an open set in $G_{i}$ that contains $p_{i}$. Hence $O_{i}=\left(\pi_{i}^{g}\right)^{-1}\left[S^{0}\left(s_{i}\right)\right]$ is open in $M^{\prime}$, and contains $p$. Suppose $p^{\prime} \in O_{i}$. Then $p^{\prime}$ lies in some element $\alpha^{\prime}=$ $\lambda\left\{s_{i}^{\prime}\right\}$ of $A$. Since $\pi_{i}^{g}\left(p^{\prime}\right) \in s_{i}^{\prime} \cap S^{9}\left(s_{i}\right), s_{i}^{\prime}$ must lie in $S\left(s_{i}\right)$, and $S\left(s_{i}^{\prime}\right) \subset T\left(s_{i}\right)$. So, by the definition of $\theta$,

$$
\theta\left(p^{\prime}\right) \subset\left(\pi_{i}^{f}\right)^{-1}\left[S\left(s^{\prime}\right)\right] \subset\left(\pi_{i}^{f}\right)^{-1}\left[T\left(s_{i}\right)\right] .
$$

Hence $\theta\left(O_{i}\right) \subset\left(\pi_{i}^{f}\right)^{-1}\left[T\left(s_{i}\right)\right]$. But $T\left(s_{i}\right) \in C_{i}(5)$, so by $\left(A^{*}\right), D\left[\theta\left(O_{i}\right)\right] \leq 2^{-i}(5 i+1)$ $<\epsilon$, for sufficiently large $i$, and $\theta$ is continuous.

To show that $\theta\left(M^{\prime}\right)=M$, let $p\left(p_{1}, p_{2}, \ldots\right) \in M$, and for each $i$, let $s_{i}$ denote an edge of $G_{i}$ containing $p_{i}$, and $\alpha_{i}$ denote an element of $A$ such that 
$\pi_{i}^{g}\left(\alpha_{i}\right)=s_{i}$. Then $\theta\left(\alpha_{i}\right) \subset\left(\pi_{i}^{f}\right)^{-1}\left[s\left(s_{i}\right)\right]$, which contains $p$ and is of diameter $\leq 2^{-i}(3 i+1)$, since $S\left(s_{i}\right) \in C_{i}(3)$. Hence the compact set $\theta\left(M^{\prime}\right)$ is dense in $M$, and $\theta\left(M^{\prime}\right)=M$.

Finally, suppose $p\left(p_{1}, p_{2}, \ldots\right) \in M$. We show that $\theta^{-1}(p)$ is an arc. Let $A_{0}$ denote the collection of all arcs of $A$ that are mapped to $p$ by $\theta$. Then $A_{0}^{*}=$ $\theta^{-1}(p)$. Now, $A_{0}$ cannot contain three arcs, $a_{j}=\lambda\left\{s_{i}^{j}\right\} \quad(j=1,2,3)$ of $A$, for suppose it does. There is a positive integer $i$ such that if $k \geq i$, then $s_{k}^{1}, s_{k}^{2}$, and $s_{k}^{3}$ are all distinct. Then by $\left(\mathrm{F}^{*}\right)$, each two of the edges $s_{i+1}^{1}, s_{i+1}^{2}$, and $s_{i+1}^{3}$ are adjacent. Hence

$$
\bigcup_{j=1}^{3} s_{i}^{j} \subset g_{i}\left[\bigcup_{j=1}^{3} s_{i+1}^{j}\right] \subset g_{i}\left[T\left(s_{i+1}^{1}\right)\right],
$$

which, by $\left(D^{*}\right)$, lies in the union of two adjacent edges of $G_{i}$. But this contradicts the fact that $s_{i}^{1}, s_{i}^{2}$, and $s_{i}^{3}$ are all distinct edges of $G_{i}$. So either $A_{0}$ contains only one arc of $A$, or only two. In the first case, $\theta^{-1}(p)$ is that arc. In the second, suppose $\alpha=\lambda\left\{s_{i}\right\} \in A$ and $\alpha^{\prime}=\lambda\left\{s_{i}^{\prime}\right\}$ are those arcs. There is a positive integer $n$ such that $s_{n} \neq s_{n}^{\prime}$. By $\left(\mathrm{F}^{*}\right), s_{n}$ and $s_{n}^{\prime}$ are adjacent edges of $G_{n}$, and by Theorem $3, \pi_{i}^{g} \mid \alpha$ and $\pi_{i}^{g} \mid \alpha^{\prime}$ are homeomorphisms onto $s_{n}$ and $s_{n}^{\prime}$, respectively. Hence $\alpha$ and $\alpha^{\prime}$ have only an endpoint in common and $\theta^{-1}(p)=$ $\alpha \cup \alpha^{\prime}$ is an arc.

\section{REFERENCES}

1. R. H. Bing, Snake-like continua, Duke Math. J. 18 (1951), 653-663. MR 13, 265.

2. M. Brown, Some applications of an approximation theorem for inverse limits, Proc. Amer. Math. Soc. 11 (1960), 478-483. MR 22 \#5959.

3. S. Eilenberg and N. E. Steenrod, Foundations of algebraic topology, Princeton Univ. Press, Princeton, N. J., 1952. MR 14, 398.

4. B. Knaster, Une continu irreductible à décomposition continue en tranches, Fund. Math. 25 (1935), 568-577.

5. S. Mardešić and J. Segal, $\epsilon$-mappings onto polyhedra, Trans. Amer. Math. Soc. 109 (1963), 146-164. MR $28 \# 1592$. 